\title{
Effects of excitotoxic striatal lesions on single unit activity in globus pallidus and entopeduncular nucleus of the cat
}

\author{
Robert N.S. Sachdev, Sid Gilman and J. Wayne Aldridge \\ Department of Neurology, University of Michigan, Ann Arbor, MI 48104 (U.S.A.)
}

(Accepted 4 April 1989)

Key words: $\gamma$-Aminobutyric acid; Single unit activity; Huntington's disease; Basal ganglia; Globus pallidus;

Entopeduncular nucleus; Partial deafferentation

\begin{abstract}
Striatal projections to the globus pallidus and entopeduncular nucleus are thought to be GABAergic and inhibitory. Thus, striatal lesions might be expected to increase the spontaneous discharge rate of neurons in these nuclei. To test this prediction, we recorded spontaneous single unit activity from awake cats sitting quietly before and 7-160 days after striatal lesions. Striatal lesions were produced by injecting ibotenic acid into the caudate nucleus and putamen. Median, standard deviation, mean, and coefficient of variation of the interspike intervals were calculated for each unit. In globus pallidus the striatal lesion resulted in a significant decrease in median interval length, i.e. an increase in the discharge rate. The prelesion median of 36 ms (S.E.M. = 2.3) decreased $11 \%$ to a postlesion value of $32 \mathrm{~ms}($ S.E.M. $=2.1)$. The lesion also resulted in a significant decrease in the variability of interspike intervals. The coefficient of variation, 1.31 (S.E.M. $=0.08$ ) before the lesion, decreased $25 \%$ to 0.97 (S.E.M. $=0.06)$ after the lesion. In entopeduncular nucleus, the lesion had no statistically significant effect on the rate of activity, but a significant decrease in the variability of activity occurred. The median interval was $33 \mathrm{~ms}(\mathrm{~S} . \mathrm{E} . \mathrm{M} .=3.3$ ) before the lesion and decreased $2 \%$ to 32 $\mathrm{ms}(\mathrm{S} . \mathrm{E} . \mathrm{M} .=2.4)$. The coefficient of variation decreased $48 \%$ from 1.44 (S.E.M. $=0.1)$ to $0.73(\mathrm{~S} . \mathrm{E} . \mathrm{M}$. $=0.03$ ). These observations support the hypothesis that loss of GABAergic inputs to the globus pallidus results in disinhibition. The discharge rate in entopeduncular nucleus was not affected by the striatal lesion, suggesting that striatal substance $\mathbf{P}$ or subthalamic excitatory inputs may have a role in regulating discharge rate in the entopeduncular nucleus.
\end{abstract}

\section{INTRODUCTION}

The striatum provides a major source of afferents to the globus pallidus (GP) and entopeduncular nucleus $(\mathrm{EPN})^{18,33,53,61}$, accounting for more than $50 \%$ of the input to the neurons of these nuclei ${ }^{21}$, 33,41 . The striatal input to both the GP and EPN contains gamma aminobutyric acid (GABA), which is thought to be inhibitory to neurons in these nuclei $^{20,39,44,51}$. Striatal inputs to the GP arise from subsets of striatal neurons separate from those to the $\mathrm{EPN}^{5}$. Striatal neurons projecting to the GP and EPN can be differentiated also by their peptidergic transmitter. Striatal substance $P$ projections selectively innervate the EPN and substantia nigra whereas striatal enkephalinergic projections innervate the $G^{2,23}$. Evidence suggests that the striatal enkephalinergic projection is inhibitory $y^{35,36}$ and the substance $P$ projection is excitatory ${ }^{14,50}$.

Striatal lesions in the rat result in a $90-95 \%$ decrease in extracellular $\mathrm{GABA}^{56}$ and a decrease in glutamic acid decarboxylase (GAD) activity in the $\mathrm{GP}$ and $\mathrm{SN}^{17,26,40}$. Striatal lesions also result in upregulation of GABA receptors in GP, EPN and $\mathrm{SN}^{42}$. Since current evidence indicates that the striatal input to GP and EPN is GABAergic and mainly inhibitory, lesions of the striatum should increase the discharge rate of neurons in GP and EPN. Surprisingly, however, following striatal ablations the discharge rate in GP and EPN decreased ${ }^{30}$. One potential explanation for the results obtained in the earlier study may be that striatal ablations remove axons of passage as well as cell bodies, thus complicating the results. In this study we produced 
striatal lesions with the axon sparing excitotoxin ibotenic acid and recorded single neuronal unit activity from GP and EPN of awake cats. Excitotoxic striatal lesions produce neuropathological and neurochemical changes similar to those seen in Huntington's disease ${ }^{7,11,63}$. Excitotoxic striatal lesions may also indicate whether neuronal discharge patterns are altered in this disease. Preliminary findings from this study have been published ${ }^{54}$.

\section{MATERIALS AND METHODS}

Four cats were habituated to the recording procedures by training them to sit quietly in a loose restraint. Two to three weeks later, they were surgically prepared for chronic single unit recording. The animals were anesthetized with pentobarbital to fix stereotaxically a chamber to the left side of the skull. The position of the chamber allowed access to the caudate nucleus, putamen, GP and EPN. One week after surgery the animals were rehabituated to the recording apparatus and exploratory recordings to locate the boundaries of the pallidum and the striatum were initiated. Data collection began 2-3 weeks after surgery. Tungsten microelectrodes stiffened by a cannula were prepared for unit recording and used in all experiments. A hydraulic microdrive was used to advance the microelectrodes to the desired recording site within the basal ganglia. Preamplified neuronal signals (gain 10,000, bandwidth $300 \mathrm{~Hz}-5 \mathrm{kHz}$ ) from the recording sites were led to an oscilloscope, audio monitor, and tape recorder. Units with initially negative peaks were isolated and unit activity was recorded on tape and digitized off-line. In two cats, one control and one lesioned, the spikes were digitized on-line ${ }^{1}$, making off-line digitization unnecessary. All units were analyzed similarly with data analysis programs prepared in our laboratory for a Compupro computer. Units recorded at a particular site within the GP or EPN were discriminated from each other and from background noise on the basis of their negative peak amplitude, positive peak amplitude, and peak to peak time $e^{60}$. Discriminated units were used for all subsequent analyses. For each unit, the mean interval length, median interval length, S.D. of the intervals, and coefficient of variation were calculated. The coefficient of variation (S.D./mean) en- abled comparisons of variability between samples of neurons with different rates.

Spontaneous unit activity was recorded from the GP and EPN of restrained cats only when they were awake and sitting quietly. Cats were trained to sit quietly during the recording session. To ensure a consistent, quiet behavioral state during recording, we observed the cats with a video camera and monitored their movements with a movement detector consisting of a high impedance cable or an accelerometer. EEG activity was recorded before and after the lesion in the first two animals to identify slow wave, large amplitude signals indicating early sleep stages. The cats rarely became drowsy during recording sessions and visual observations proved as useful as EEG, therefore, subsequent cats were monitored with a video camera alone. Units recorded during drowsiness were not used for analysis. Segments of unit activity recorded during movement as indicated by direct observation or by the movement detector were also not used for analysis.

The first two cats were studied both before and after the lesion. Before the lesion, penetrations through GP and EPN were made through alternating tracks in a checkerboard fashion. The remaining tracks, neighboring those recorded prior to the lesion, were studied after the lesion. This procedure ensures that the recording sites within the GP and EPN before and after the striatal lesion are similar. One additional cat was studied as a non-lesioned control and another was studied only after the lesion.

Unilateral striatal lesions were produced by multiple injections of ibotenic acid $(2 \mu \mathrm{g} / 200 \mu \mathrm{l}$ phosphate buffer solution) into the caudate nucleus and putamen. Eleven to 19 injection sites ranging from $0.25-1.5 \mu \mathrm{l} / \mathrm{site}$ were used to produce the striatal lesions. To avoid unintentional damage to the GP, smaller amounts of ibotenic acid were injected into the putamen (3-5 injection sites) as compared to the caudate nucleus (8-15 injection sites). Histologic reconstructions of the recording sites were facilitated by marking 6 recording tracks with deposits of iron on the final day of recording. Direct current $(100 \mu \mathrm{A}$ for 30-45 s) was passed through a bipolar steel electrode providing iron deposits that were stained blue by adding $1.5 \%$ potassium ferrocyanide to the 
$10 \%$ formalin perfusing solution. Animals were perfused trans-cardially under deep barbiturate anesthesia. The brains were removed, postfixed in sucrose/formalin solution, frozen, sectioned, and stained with Cresyl violet. The recording tracks and lesions were reconstructed and drawn on maps traced from stained sections. An image analysis computer was used to determine the lesion size. The area of the striatum on the lesioned and control sides of each coronal section were computed by interactively tracing the striatal boundaries on digitized images. Reconstructed tracks from all animals are shown in Fig. 3. Snider and Niemer's stereotaxic atlas of the cat brain ${ }^{57}$ was used as a guide in reconstruction and in preparing Fig. 2.

The Mann-Whitney $U$ test and the $\chi^{2}$ test were used to assess the significance of changes in unit activity.

\section{RESULTS}

\section{Histology}

In all animals the lesion included the rostro-lateral and the caudo-lateral regions of the head and body of the caudate nucleus (Fig. 1A). Lesions within the putamen were restricted to the rostro-lateral regions and were smaller than lesions of the caudate nucleus (Fig. 1). The lesions produced a 30 to $50 \%$ loss of striatal tissue (Fig. 2). The lateral ventricle ipsilateral to the lesion was enlarged and gliosis was found at the site of the lesion (Fig. 1). The lateral regions of the caudate nucleus were heavily damaged while some medial regions were spared (Fig. 1). In one cat, small lesions of the rostral claustrum adjacent to the putamen were observed. No damage to any other anatomical structure was noted.
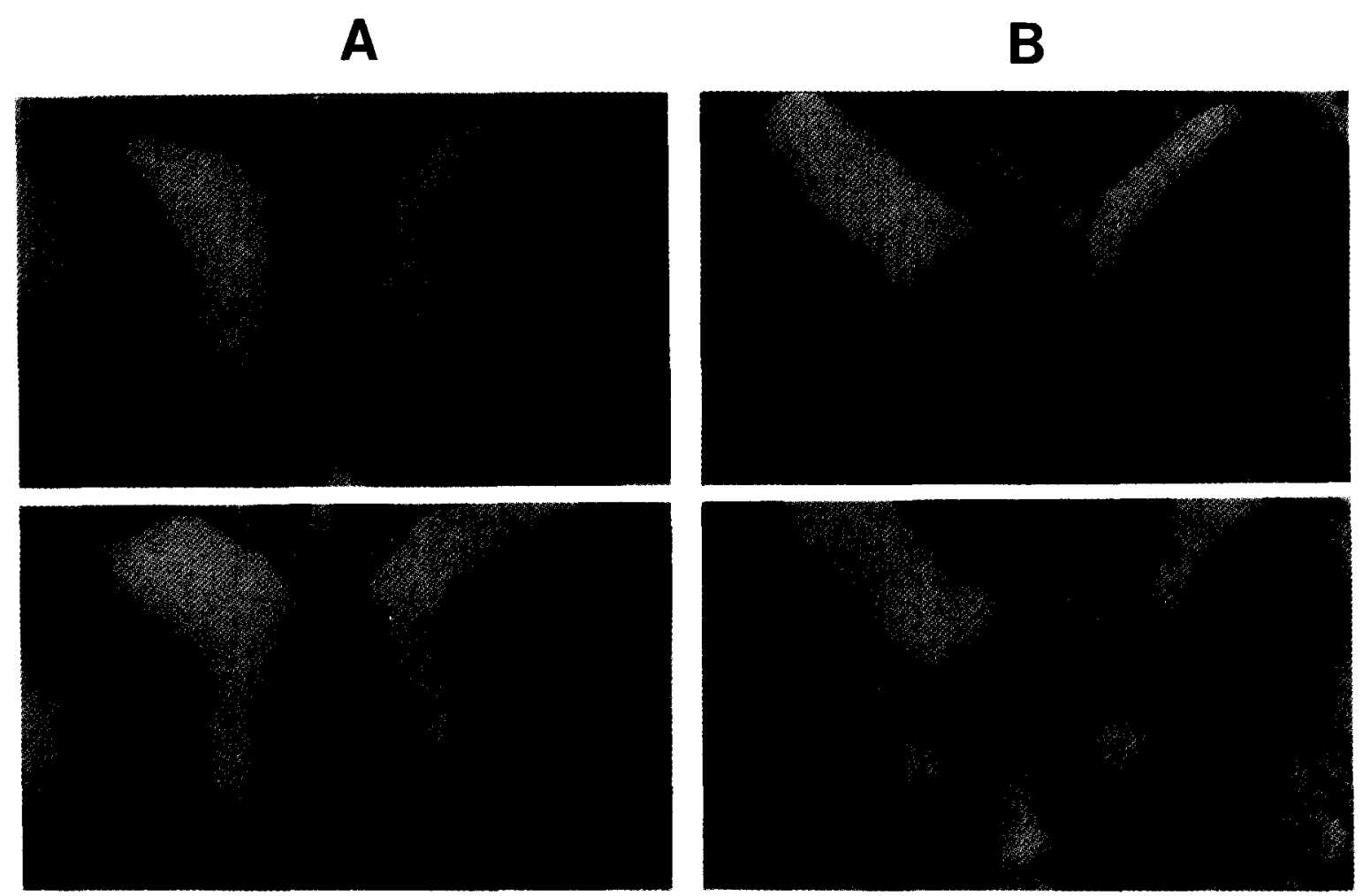

Fig. 1. Photomicrographs of Cresyl violet stained tissue sections illustrating the striatal lesions in two cats. A: the upper section is from the rostral extent of the caudate nucleus. The lower section is from a more caudal level in the same animal. B: in a second animal, the upper section is more rostral than the lower section. Note that in both animals the lesion affects the lateral regions of the caudate nucleus more than the medial regions. 


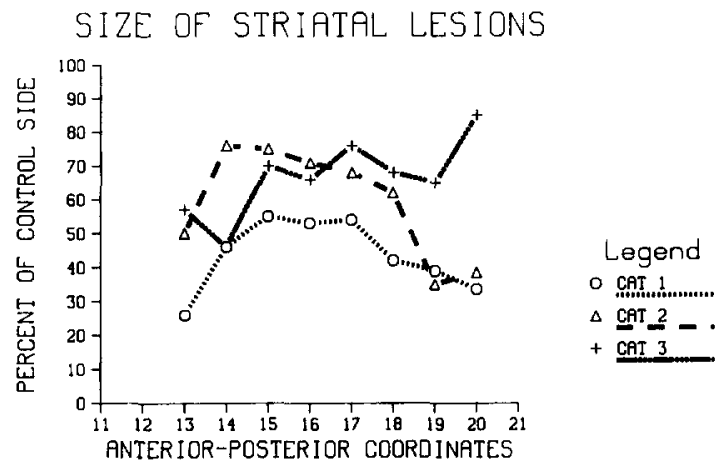

Fig. 2. Lesion size. The striatal area on the lesioned side was computed as a percentage of the striatal area on the control side ( $y$-axis), and is plotted as a function of the anteriorposterior coordinates ( $x$-axis).

\section{Site of recording}

In all animals, recordings were taken from similar sites in the GP and EPN before and after the lesion (Fig. 3).

\section{Globus pallidus}

Unit activity was recorded from the GP between 7 and 160 days after the lesion. Four hundred fifty seven units were recorded. Of this total, 192 units were recorded prior to the lesion and 265 units after the lesion. The lesion resulted in a significant decrease in the median length of the interspike intervals (Table I). This decrease was due to the presence of an increased proportion of units with short median intervals (Fig. 4A,B). The lesion altered the variability of the unit activity in GP. The average coefficient of variation decreased by $25 \%$ after the lesion (Table 1). The decrease in the coefficient of variation resulted from an increase in the proportion of units with small coefficients of variation (Fig. 4C,D).

The decrease in median interval was accompanied by a significant $\left(\chi^{2}\right.$ test $\left.P<0.01\right)$ decrease in the proportion of units containing long (>500 ms) interspike intervals (Fig. 6A,B). Units with at least
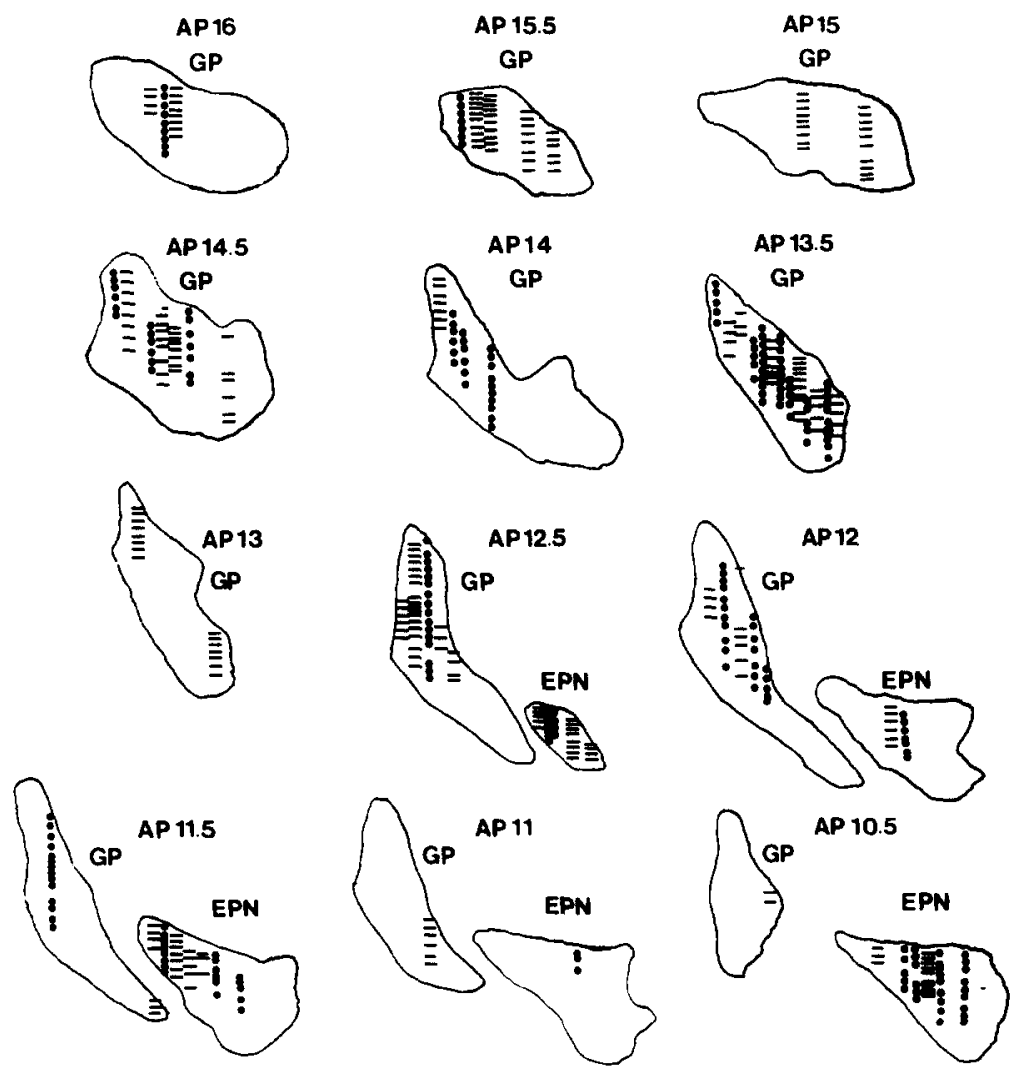

Fig. 3. Representation of all recording sites in all animals studied. Prelesion recording sites are plotted as filled circles and postlesion recording sites are plotted as dashes. 
TABLE I

Globus pallidus and entopeduncular nucleus unit activity

\begin{tabular}{|c|c|c|c|c|c|}
\hline \multirow[b]{3}{*}{ Median ISI (ms) } & \multicolumn{2}{|c|}{ Globus pallidus } & \multicolumn{3}{|c|}{ Entopeduncular nucleus } \\
\hline & Control & Lesion & Control & \multicolumn{2}{|c|}{ Lesion } \\
\hline & 36 & $32 \quad(2.1)^{* *}$ & $33 \quad(3.3)$ & 32 & (2.4) \\
\hline Mean ISI (ms) & $63 \quad(5.3)$ & $43 \quad(2.9)^{* * *}$ & $55 \quad(5.7)$ & 46 & (4.5) \\
\hline Coefficient of variation & $1.30(0.08)$ & $0.98(0.03)^{*}$ & $1.42(0.1)$ & 0.74 & $(0.03)^{* * *}$ \\
\hline Standard deviation (ms) & 111 (16.7) & $41 \quad(2.8)^{* * *}$ & $79 \quad(9.9)$ & 34 & $(5.3)^{* * * *}$ \\
\hline Number of units & 192 & 265 & 65 & 70 & \\
\hline
\end{tabular}

${ }^{* * *} P<0.0001 ;{ }^{* *} P<0.001 ;{ }^{*} P<0.05$ as assessed by the Mann-Whitney U test; ISI $=$ interspike interval; ms $=$ milliseconds; numbers in brackets represent standard errors.

one interval greater than $500 \mathrm{~ms}$ were compared to units that had no intervals greater than $500 \mathrm{~ms}$. Both before and after the striatal lesion, units with at least one interspike interval greater than $500 \mathrm{~ms}$ had significantly larger (Mann-Whitney $\mathrm{U}$ test $P<$ $0.0001)$ median intervals and higher coefficients of variation (Mann-Whitney $U$ test $P<0.0001$ ) than units without long intervals (Table II).

\section{Entopeduncular nucleus}

Unit activity in EPN was recorded 25-145 days after the lesion. A total of 135 units was recorded,
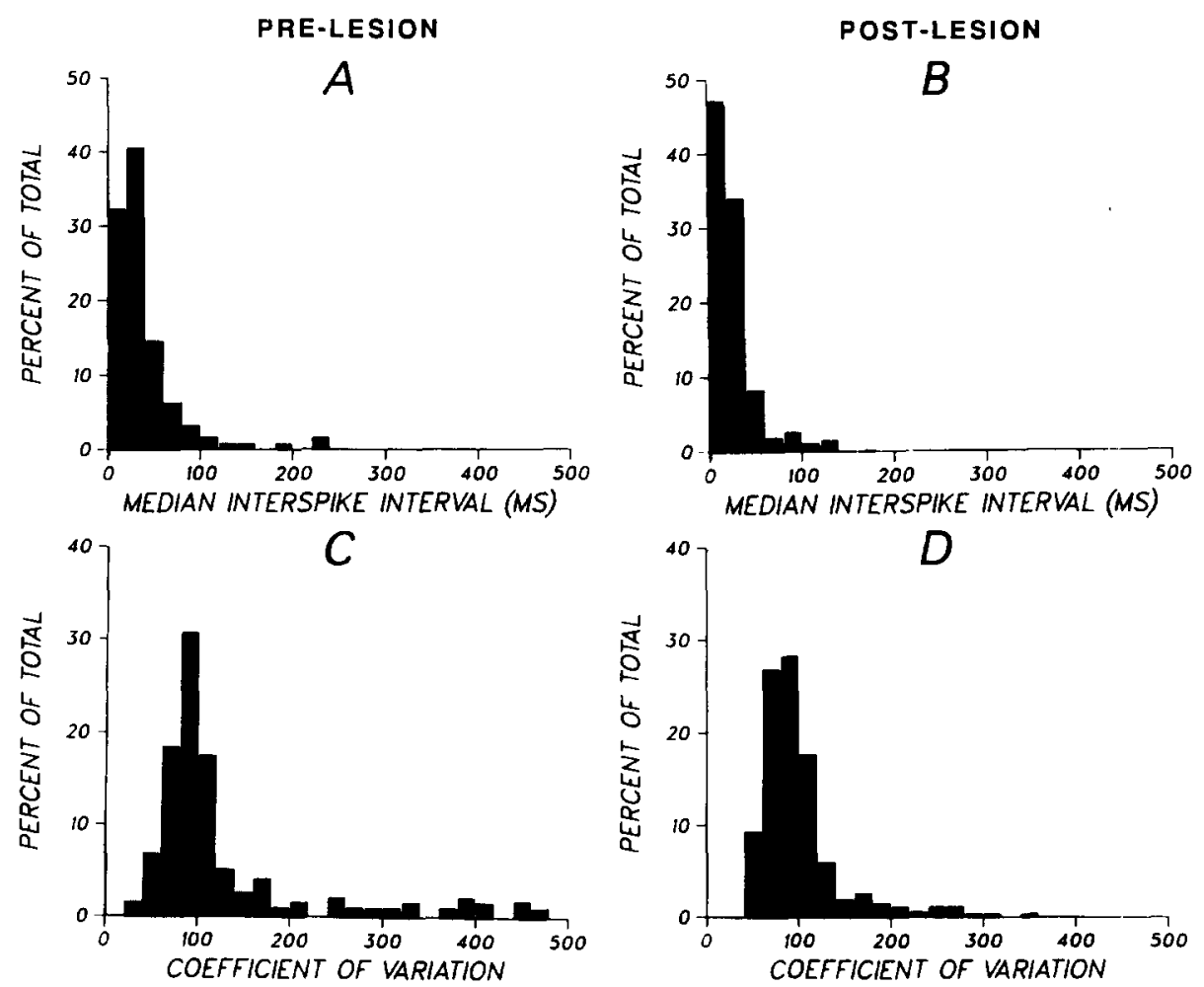

Fig. 4. Distributions of median intervals and coefficients of variation of all units in the globus pallidus. A: prelesion and B: postlesion distributions of median intervals. C: prelesion, and D: postlesion distributions of coefficients of variation. Note that the postlesion distributions of the median intervals and coefficients of variation are shifted to the right, however, the prelesion and postlesion distributions overlap. 
TABLE II

Globus pallidus units containing long intervals

\begin{tabular}{|c|c|c|c|c|}
\hline \multirow[b]{3}{*}{ Median ISI (ms) } & \multicolumn{2}{|l|}{ Control } & \multicolumn{2}{|l|}{ Lesion } \\
\hline & $I S I>500 \mathrm{~ms}$ & $I S I<500 \mathrm{~ms}$ & $I S I>500 \mathrm{~ms}$ & $I S I<500 \mathrm{~ms}$ \\
\hline & $51 \quad(5.4)$ & $27(1.4)^{*}$ & $61 \quad(8.5)$ & $24 \quad(1.0)^{*}$ \\
\hline Mean ISI (ms) & $112(12.3)$ & $34 \quad(1.6)^{*}$ & $91 \quad(11.1)$ & $31 \quad(1.3)^{*}$ \\
\hline Coefficient of variation & $2.12(0.17)$ & $0.83(0.01)^{*}$ & $1.50(0.09)$ & $0.85(0.02)^{*}$ \\
\hline Standard deviation (ms) & $255 \quad(46.6)$ & $28 \quad(1.4)^{*}$ & $101 \quad(9.8)$ & $26(1.16)^{*}$ \\
\hline Number of units ( $\%$ of total) & $70 \quad(37 \%)$ & $122(63 \%)$ & $53(19 \%)$ & $212(81 \%)$ \\
\hline
\end{tabular}

${ }^{*} P<0.0001$ as assessed by the Mann-Whitney U test. Comparisons between ISI $>500 \mathrm{~ms}$ and ISI $<500 \mathrm{~ms}$; ISI $=$ interspike interval; $\mathrm{ms}=$ milliseconds; numbers in brackets represent standard errors.

65 prior to the lesion and 70 after the lesion. The lesion resulted in a non-significant $2 \%$ decrease in median interspike interval (Table I). The distribution of median intervals in EPN is shown in Fig. $5 A, B$. Although the median rates of activity were unaffected by the lesion, the proportion of spiketrains with long intervals decreased significantly $\left(\chi^{2}\right.$
$P<0.01$ ) (Fig. 6). The lesion had a marked effect on the variability of activity in EPN. The coefficient of variation decreased by $48 \%$ as compared to prelesion values (Table I). The decrease in the coefficient of variation is related to an increase in the proportion of units with small coefficients of variation (Fig. 5C,D).
PRE-LESION
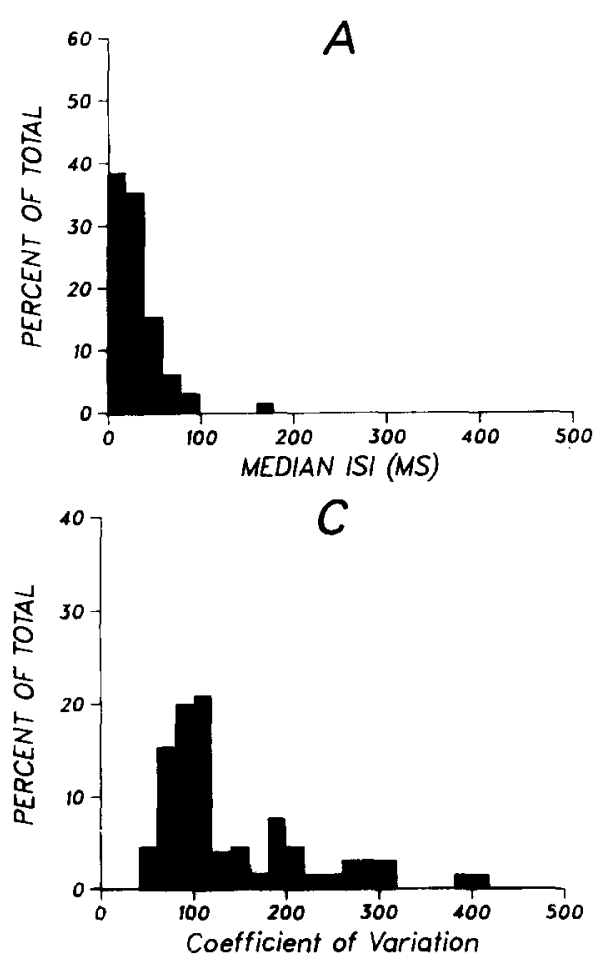

POST-LESION
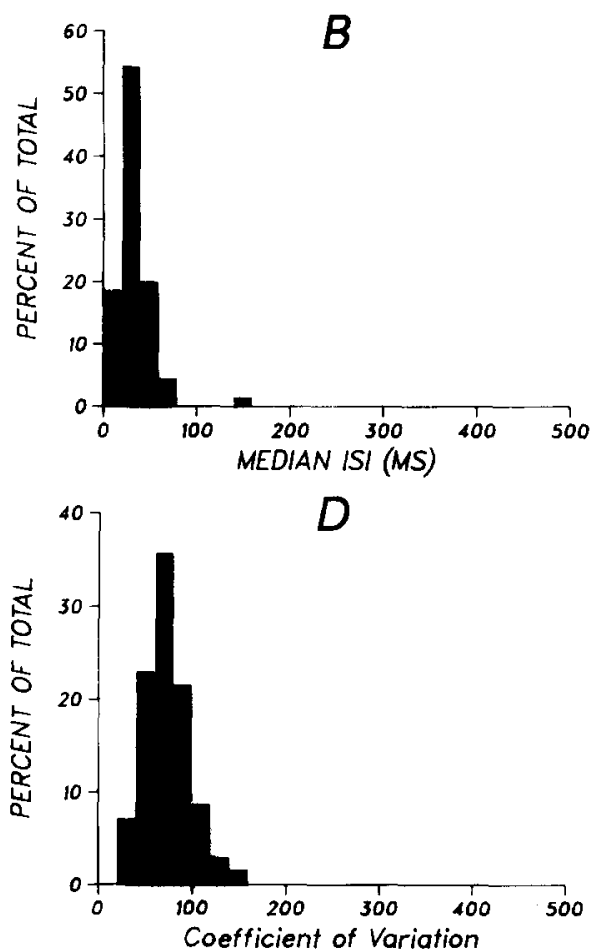

Fig. 5. Distributions of the median intervals and coefficients of variation of all units in the entopeduncular nucleus. A: prelesion and B: postlesion distribution of median intervals. C: prelesion and D: postlesion distributions of coefficients of variation. Note that the distribution of median intervals is unaffected by the lesion, but the distribution of coefficients of variation is shifted to the right. 
PRE-LESION
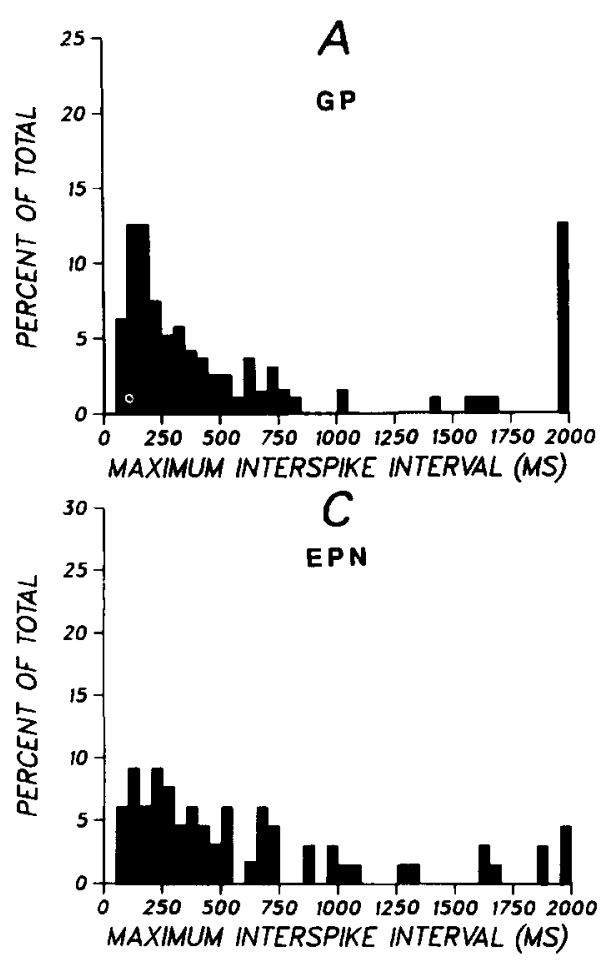

POST-LESION
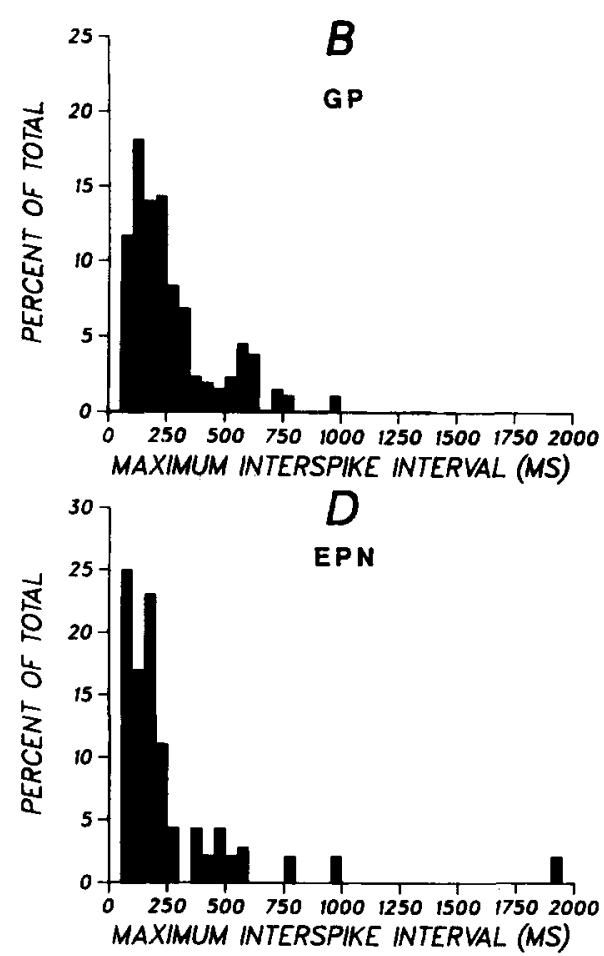

Fig. 6. Distributions of the largest interspike intervals of all cells in each spiketrain recorded from the globus pallidus and entopeduncular nucleus. A: prelesion and B: postlesion distributions in globus pallidus. C: prelesion and D: postlesion distribution in the entopeduncular nucleus. Note that the spiketrains in both globus pallidus and entopeduncular nucleus have a similar proportion of large ( $>500 \mathrm{~ms})$ intervals, both before and after the striatal lesion.

As in the GP, the subgroup of units with at least one interval greater than $500 \mathrm{~ms}$ had, on average, longer median intervals (Mann-Whitney U test $P<$ 0.05 ) and higher coefficients of variation (MannWhitney $U$ test $P<0.001$ ) than units with no intervals greater than $500 \mathrm{~ms}$ (Table III).

\section{Topography}

Units in GP were grouped according to their anatomical location. The GP was arbitrarily divided into 4 anatomical quadrants ${ }^{43}$ : rostro-medial (anterior-posterior $(\mathrm{AP})$ coordinates $\geqslant 13.5$ and lateral (L) coordinates $\leqslant 7.5)$, rostro-lateral $(A P \geqslant 13.5, \mathrm{~L}$ $>7.5)$, caudo-medial $(\mathrm{AP}<13.5, \mathrm{~L}<9)$ and

TABLE III

Entopeduncular nucleus units containing long intervals

\begin{tabular}{|c|c|c|c|c|c|}
\hline \multirow[b]{3}{*}{ Median ISI (ms) } & \multicolumn{2}{|l|}{ Control } & \multicolumn{3}{|l|}{ Lesion } \\
\hline & $I S I>500 \mathrm{~ms}$ & $I S I<500 \mathrm{~ms}$ & $I S I>500 \mathrm{~ms}$ & \multicolumn{2}{|c|}{$I S I<500 \mathrm{~ms}$} \\
\hline & $43 \quad(6.6)$ & $26(2.3)^{*}$ & $61 \quad(17.1)$ & 29 & $(1.7)$ \\
\hline Mean ISI (ms) & $82 \quad(10.8)$ & $34(2.8)^{*}$ & $111(38.4)$ & 35 & $(2.3)$ \\
\hline Coefficient of variation & $1.93(0.16)$ & $1.04(0.9)^{*}$ & $1.23(0.09)$ & 0.69 & $(0.02)$ \\
\hline Standard deviation (ms) & $140(17.2)$ & $34 \quad(2.9)^{*}$ & 131 & 25 & $(2.3)$ \\
\hline Number of units (\% of total) & $28 \quad(43 \%)$ & $37 \quad(57 \%)$ & $6 \quad(7 \%)$ & 64 & $(93 \%)$ \\
\hline
\end{tabular}

${ }^{*} P<0.0001$ as assessed by the Mann-Whitney U test. Comparisons between ISI $>500 \mathrm{~ms}$ and ISI $<500 \mathrm{~ms}$; ISI $=$ interspike interval; $\mathrm{ms}=$ milliseconds; numbers in brackets represent standard errors. 

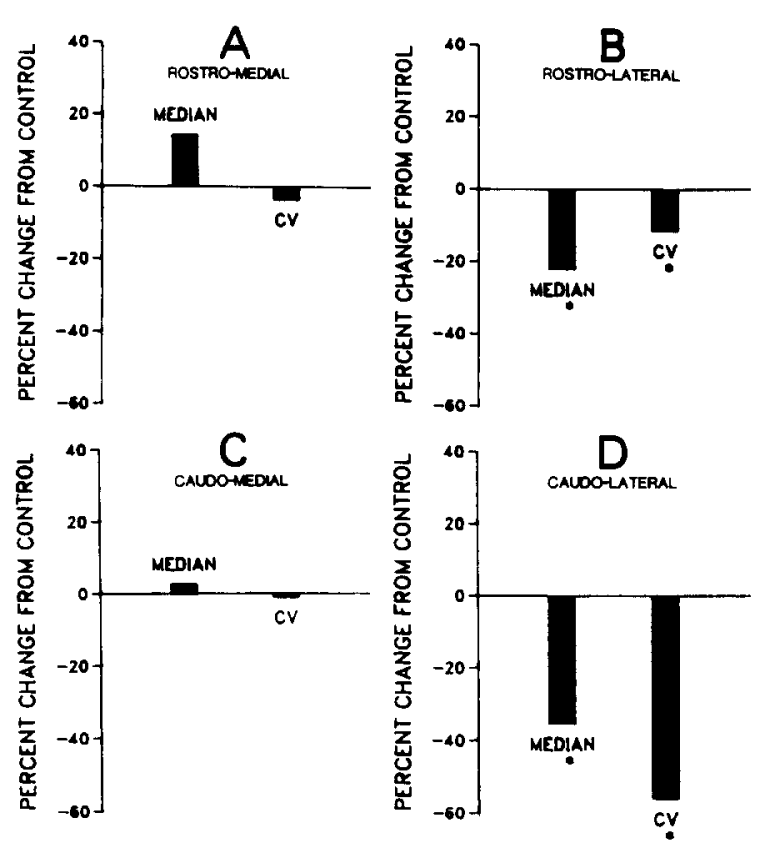

Fig. 7. Percentage change in the median intervals and coefficients of variation in each quadrant of the globus pallidus. A: rostro-medial quadrant (anterior-posterior (AP) coordinates $\geqslant 13.5$ and lateral $(\mathrm{L})$ coordinates $\leqslant 7.5$ ). $\mathrm{B}$ : rostro-lateral quadrant $(\mathrm{AP} \geqslant 13.5$ and $\mathrm{L}>7.5$ ). $\mathrm{C}$ : caudo-medial quadrant $(A P<13.5, L \leqslant 9)$. D: caudo-lateral quadrant $(\mathrm{AP}<13.5$, $\mathrm{L}>9$ ). The bins with * represent significant changes in the median intervals or coefficients of variation following the lesion.

caudo-lateral (AP $<13.5, L \geqslant 9$ ). Units collected from each of these quadrants were grouped together and statistics for each group were calculated. The analysis indicated that the lesion affected the median

\section{CAUDO-LATERAL OUADRANT: RECOVERY}

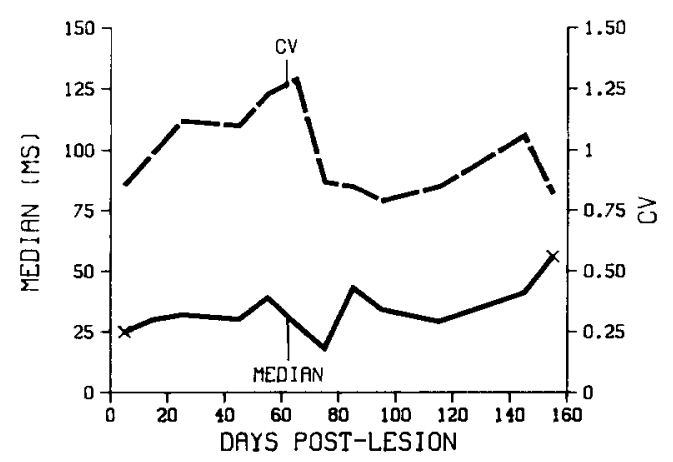

Fig. 8. Graph of median intervals and coefficients of variation in the 160 days after the striatal lesion in the caudo-lateral quadrant of the globus pallidus only. Note that no consistent trend towards recovery occurs for any of the statistics. and coefficient of variation in the lateral quadrants of GP alone (Fig. 7B,D). The medial quadrants of GP had similar medians and coefficients of variation both before and after the lesion (Fig. 6A,C). Topographic differences in the rate and pattern of activity in EPN were examined but no significant differences between anterior-posterior or mediolateral quadrants were demonstrable.

\section{Recovery}

Trends towards recovery in the rate and variability of interspike intervals in both GP and EPN were assessed by examining changes in data obtained over the months following the lesion. After the lesion, data from successive 10-day periods were culled and the median, mean, S.D., and coefficient of variation from each 10-day period were compared. No consistent trend towards recovery was demonstrable for any of the statistics in either GP or EPN. Data recorded from the caudo-lateral quadrant of GP, the quadrant most affected by the lesion, are illustrated in Fig. 8.

\section{DISCUSSION}

This study demonstrated that excitotoxic injury of the striatum alters spontaneous neuronal activity in the GP and EPN. The lesion resulted in an increase in the discharge rate in the GP but not in the EPN and a change in the variability of interspike intervals in both nuclei.

The changes in spontaneous unit activity of deafferented GP neurons are consistent with the loss of a tonic inhibitory input from the striatum. The lesion resulted in a significant decrease in median interval, a decrease in the variability of discharge and a decrease in the proportion of units containing long intervals. The decrease in median interval, i.e. an increase in firing rate, is the change expected from loss of tonic inhibition. The decrease in variability of firing is related to the decreased proportion of slowly firing neurons, i.e., those containing long intervals. The decrease in variability may thus be related to loss of GABAergic striatal input, the decrease in long intervals resulting from a decrease in long lasting striatal inhibition ${ }^{44}$. GABA is an inhibitory neurotransmitter and enkephalin, the peptidergic neurotransmitter colocalized with 
GABA in the striatal projections to GP, is thought to be inhibitory as well ${ }^{36,37,48}$.

Excitotoxic injury of the striatum had no effect on the discharge rate in EPN. This may be related to the effects of substance $P$, a peptidergic neurotransmitter present in the striatal projections to the EPN ${ }^{2.23 .48}$. Substance $P$ is thought to be excitatory ${ }^{14.50}$ and its absence following the striatal lesion may counteract the loss of inhibitory GABAergic projections to EPN. The interactive effects of the loss of two transmitters may explain the lack of a change in rate, but it does not explain the change in variability or the change in the proportion of long intervals. An explanation for the decrease in variability and the decrease in the proportion of long intervals in EPN may be that GABA release in the EPN normally results in long intervals and therefore more variability. The loss of striatal GABAergic inputs to the EPN therefore reduces the long intervals and the variability of activity in EPN. Thus, the striatal lesion results in the loss of two inhibitory transmitters in GP and loss of one inhibitory and one excitatory transmitter in EPN. This difference may explain the different effects of the striatal lesion, upon GP and EPN.

Other inputs to GP and EPN may also be affected by the striatal lesion and contribute to the altered discharge rates and variability. It is likely that the striatal lesion indirectly affects subthalamic inputs to the GP and EPN. The majority of excitatory inputs to GP and EPN are from the subthalamic nucleus and this nucleus in turn receives inhibitory inputs from $\mathrm{GP}^{9.10,34.37}$. The increased discharge rate in GP presumably increases inhibitory input to the subthalamic nucleus, thus causing subthalamic neurons to discharge more slowly. Slower discharge in subthalamus would reduce the excitatory drive impinging on GP and EPN neurons, thereby further reducing the discharge rate in these nuclei. However, since there is no evidence that subthalamic inputs have different effects on GP than on EPN neurons, it is unlikely that these inputs are responsible for the different effects of the striatal lesion on the GP and EPN discharge rate. The substantia nigra, another input to the GP and EPN, may also be affected by the striatal lesion ${ }^{43}$. The substantia nigra receives direct inhibitory projections from the striatum and may, therefore, discharge faster after the lesion ${ }^{43}$.
However, there is no evidence that the substantia nigra projections to GP are different from those to EPN. Therefore, increased discharge rate in substantia nigra cannot account for differential effects of the striatal lesion on GP and EPN discharge rate.

Alteration of neuronal membrane properties may explain some of the changes in neuronal discharge rate and variability of firing. Neuronal morphology affects passive membrane properties ${ }^{28,29}$ and in many systems, including the basal ganglia, neurons that are deafferented undergo morphological reorganization $^{45,58}$. It is possible that the effects of the striatal lesion on the variability and rate of activity could be due, at least in part, to changes in morphology. There is, however, no reason to expect that the morphological changes in EPN would be different than the morphological changes in GP. Thus, changes in morphology following the striatal lesion would not explain the different effects of the striatal lesion on the rate of discharge in GP and EPN.

An earlier study on the effects of striatal lesions on GP and EPN disclosed a decrease in the rate of activity in these structures ${ }^{30}$. Methodologic factors may account for the differences in results between the previous study and the present one. In the earlier experiments, ablations by suction were used to produce striatal lesions. This type of ablation interrupts fibers of passage in addition to lesioning cell bodies. In the present study the axon sparing excitotoxin ibotenic acid was used to produce the striatal lesions. In the earlier study, lesions were restricted to the caudate nucleus whereas in the present study the caudate nucleus and parts of the putamen were lesioned. Finally, in the earlier study the animals were acutely prepared cats relaxed with gallamine and locally anesthetized and in the present study the animals were chronically prepared and loosely restrained.

Spontaneous unit activity recorded in the lateral and medial segments of the GP in the primate differs from spontaneous unit activity recorded in the GP and EPN of the cat. In the primate, within the lateral segment of the GP, which is equivalent to the GP in the cat, units with at least one long interval (one interval $>500 \mathrm{~ms}$ ) constitute about $85 \%$ of the units $^{15,16,19}$. In the cat GP, long intervals are present in only $37 \%$ of the units. The primate medial 
segment of GP, which is equivalent to the cat EPN, has no units containing long intervals. In the EPN of the cat, $43 \%$ of the spiketrains contain long intervals. This difference in the proportion of units containing long intervals suggests that the criteria used to distinguish 3 patterns of activity in the primate pallidum ${ }^{15,16,19}$ cannot be used in the cat pallidum. It is possible that anatomical differences between species such as the presence or absence of recurrent axons ${ }^{61}$ may account for these differences in unit activity.

Excitotoxic striatal lesions altered unit activity in the lateral regions but not the medial regions of the GP. These findings are consistent with the topographic distribution of projections from the striatum to the GP and with the locations of the lesions placed in the striatum ${ }^{38,53}$. The lateral regions of the caudate nucleus project to the lateral regions of the $\mathrm{GP}^{53}$. Moreover, the projections from the caudate nucleus to the GP and EPN maintain a mediolateral, dorsoventral and anteroposterior topographic correspondence ${ }^{53}$. The lesions in our animals were larger in the lateral than the medial parts of the striatum and thus the lateral regions of both the GP and EPN are more likely to have been affected by the lesion than the medial regions. The fact that striatal projections to both GP and EPN are similarly organized topographically ${ }^{53}$ indicates that the different effects of the striatal lesion upon these structures in the present study do not result from selective deafferentation of only one of these structures.

The discharge rate of a neuron is one mechanism for encoding and transmitting information in neuronal systems ${ }^{8,49}$. The discharge rate can potentially determine: (a) the type of transmitter released by a neuron $^{4,32}$; (b) the amount of transmitter released ${ }^{3}$, ${ }^{31}$; and (c) whether long-term potentiation occurs ${ }^{6}$.

\section{REFERENCES}

1 Aldridge, J.W., Walden, J.L. and Gilman, S., Enhancing high-speed digitization of single unit neuronal activity on a microcomputer using a hybrid software-hardware technique, $J$. Neurosci. Meth., in press.

2 Aronin, N., DiFiglia, M., Graveland, G.A., Schwartz, W.J. and $\mathrm{Wu}, \mathrm{J}$, , Localization of immunoreactive enkephalins in GABA synthesizing neurons of the rat neostriatum, Brain Research, 300 (1984) 376-380.

3 Augustine, G.J., Charlton, M.P. and Smith, S.J., Calcium action in synaptic transmitter release, Ann. Rev. Neurosci., 10 (1987) 633-693.
Changes in the discharge rate of GP neurons following striatal lesions may alter the information transmitted between GP and its target sites and this could lead to changes in biochemical, morphological, pharmacological or gene expression of the pallidal targets ${ }^{22,62}$. Rate is unlikely to be the sole determinant of information transfer. The rate of activity in EPN is unaffected by the striatal lesion, but other studies of EPN target regions (VA-VL thalamus) demonstrate changes following striatal lesions ${ }^{25,27,42}$. These results suggest that the variability of activity may be an important means of encoding information as well.

Hypotheses attempting to explain the mechanisms underlying the chorea ${ }^{12,13,46,47,52}$ or bradykinesia ${ }^{59}$ of Huntington's disease have focused on the effects of striatal lesions on the discharge rates in pallidum, VA/VL thalamus and cortex. Our results suggest that changes in variability and burst discharges ${ }^{55}$ may also be important.

\section{ACKNOWLEDGEMENTS}

The authors gratefully acknowledge the technical assistance provided by $\mathbf{M}$. Gallagher, D. Jaeger, $\mathbf{M}$. Lesperance, Dr. J.B. Penny, S. Ruger, and J. Walden. Helpful comments were provided by $D$. Jaeger, R. Marcus, and J. Walden. The authors are grateful to Drs. R. Albin, R.L. Macdonald, S. Winans Newman, and A.B. Young for their helpful suggestions. This work was supported in part by NIH Grants NS 19613, NS 07222, a grant from the Hereditary Disease Foundation, two fellowships awarded by the University of Michigan Medical School (to R.N.S.S.), and a grant from the United Cerebral Palsy Research and Education Foundation, Inc.

4 Bartfai, T., Iverfeldt, K., Brodin, E. and Ogren, S. Functional consequences of coexistence of classical and peptide neurotransmitters, Prog. Brain Res., 68 (1986) 331-335.

5 Beckstead, R.M. and Cruz, C.J., Striatal axons to the globus pallidus, entopeduncular nucleus and substantia nigra come mainly from separate cell populations in cat, Neuroscience, 19 (1986) 147-158.

6 Bliss, T.V.P. and Lømo, T., Long-lasting potentiation of synaptic transmission in dentate area of anesthetized rabbit following stimulation of perforant path, J. Physiol. (Lond.), 232 (1973) 357-374.

7 Bruyn, G.W., Huntington's chorea: historical, clinical and 
laboratory synopsis. In P. Vinken and G.W. Bruyn (Eds.), Handbook of Clinical Neurology, Vol. 6, North Holland Publishing Co., Amsterdam, 1968, pp. 298-378.

8 Bullock, T.H., Orkland, R. and Grinnell, A., Introduction to Nervous Systems, W.H. Freeman and Company, San Francisco, 1977, 559 pp.

9 Carpenter, M.B., Batton III, R.R., Carleton, S.C. and Keller, J.T., Interconnections and organization of pallidal and subthalamic nucleus neurons in the monkey, J. Comp. Neurol., 197 (1981) 579-609.

10 Carpenter, M.B., Carleton, S.C., Keller, J.T. and Conte, P., Connections of the subthalamic nucleus in the monkey, Brain Research, 224 (1981) 1-29.

11 Coyle, J.T. and Schwarcz, R., Lesion of striatal neurons with kainic acid provides a model for Huntington's chorea, Nature (Lond.), 263 (1976) 244-246.

12 Crossman, A.R., Mitchell, I.J., Sambrook, M.A. and Jackson, A., Chorea and myoclonus in the monkey induced by gamma-aminobutyric acid antagonism in the lentiform complex, Brain, 111 (1988) 1211-1233.

13 Crossman, A.R., Sambrook, M.A. and Jackson, A., Experimental hemichorea/hemiballismus in the monkey: studies on the intracerebral site of action in a drug-induced dyskinesia, Brain, 107 (1984) 479-596.

14 Davies, J. and Dray, A., Substance P in the substantia nigra, Brain Research, 107 (1976) 623-627.

15 DeLong, M.R., Activity of pallidal neurons during movement, J. Neurophysiol., 34 (1971) 414-427.

16 DeLong, M.R., Crutcher, M.D. and Georgopoulos, A.P., Primate globus pallidus and subthalamic nucleus: functional organization, J. Neurophysiol., 53 (1985) 530-543.

17 DiChiara, G., Morelli, M., Porceddu, M.L., Mulas, M. and Del Fiacco, M., Effect of discrete kainic acid-induced lesions of corpus caudatus and globus pallidus on glutamic acid decarboxylase of rat substantia nigra, Brain Research, 189 (1980) 193-208.

18 DiFiglia, M., Pasik, P. and Pasik, T., A Golgi and ultrastructural study of the monkey globus pallidus, $J$. Comp. Neurol., 212 (1982) 53-75.

19 Fillion, M., Effects of interruption of the nigrostriatal pathway and of dopaminergic agents on the spontaneous activity of globus pallidus neurons in the awake monkey, Brain Research, 178 (1979) 425-441.

20 Fonnum, F., Gottesfeld, Z. and Grofová, I., Distribution of glutamate decarboxylase, choline acetyltransferase and aromatic amino acid decarboxylase in the basal ganglia of normal and operated rats. Evidence for striatopallidal, striatoentopeduncular and striatonigral GABAergic fibres, Brain Research, 143 (1978) 125-138.

21 Fox, C.A., Andrade, A.N., LuQui, I.J. and Rafoals, J.A., The primate globus pallidus: a Golgi and electron microscopic study, J. Hirnforsch., 15 (1974) 75-93.

22 Francois, C., Percheron, G., Yelnick, J. and Heyner, S., A Golgi analysis of the primate globus pallidus. I. Inconstant processes of large neurons, other neuronal types, and afferent axons, J. Comp. Neurol., 227 (1984) 182-199.

23 Groenewegen, H.J. and Russchen, F.T., Organization of the efferent projections of the nucleus accumbens to pallidal, hypothalamic, and mesencephalic structures: a tracing and immunohistochemical study in the cat, $J$. Comp. Neurol., 223 (1984) 347-367.

24 Hendry, S.H.C. and Jones, E.G., Reduction in number of immunostained GABAergic neurons in deprived-eye dom- inance columns of monkey area 17, Nature (Lond.), 320 (1986) $750-753$.

25 Hosokawa, S., Kato, M., Shima, F., Tobimatsu, S. and Kuroiwa, Y., Local cerebral glucose utilization altered in rats with unilateral electrolytic striatal lesions and modification by apomorphine, Brain Research, 324 (1984) 59-68.

26 Isacson, O., Brundin, P., Gage, F.H. and Björklund, A., Neural grafting in a rat model of Huntington's disease: progressive neurochemical changes after neostriatal ibotenate lesions and striatal tissue grafting, Neuroscience, 16 (1985) 799-817.

27 Kelly, P.A.T., Graham, D.I. and McCulloch, J., Specific alterations in local cerebral glucose utilization following striatal lesions, Brain Research, 233 (1982) 157-172.

28 Koch, C., Poggio, T. and Torre, V., Nonlinear interactions in a dendritic tree: localization, timing, and role in information processing, Proc. Natl. Acad. Sci. U.S.A., 80 (1983) 2799-2802.

29 Koch, C., Poggio, T. and Torre, V., Retinal ganglion cells: a functional interpretation of dendritic morphology, Phil. Trans. R. Soc. Lond. B, 298 (1982) 227-264.

30 Levine, M.S., Hull, C.D., Villablanca, J.R., Buchwald, N.A. and Garcia-Rill, E., Effects of caudate nuclear or frontal cortical ablation in neonatal kittens or adult cats on the spontaneous firing of forebrain neurons, Dev. Brain Res., 4 (1982) 129-138.

31 Llinás, R., Steinberg, I.Z. and Walton, K., Relationship between presynaptic calcium current and postsynaptic potential in squid giant synapse, Biophys. J., 33 (1981) 323-352.

32 Lundberg, J.M. and Hökfelt, T., Multiple co-existence of peptides and classical transmitters in peripheral autonomic and sensory neurons - functional and pharmacologic implications, Prog. Brain Res., 68 (1986) 241-262.

33 Moriizumi, T., Nakamura, Y., Okoyama S. and Kitao, Y., Synaptic organization of the cat entopeduncular nucleus with special reference to the relationship between the afferents to entopedunculothalamic projection neurons: an electron microscope study by a combined degeneration and horseradish peroxidase tracing technique, Neuroscience, 20 (1987) 797-816.

34 Nakanishi, H., Kita, H. and Kitai, S.T., Intracellular study of rat substantia nigra pars reticulata neurons in an in vitro slice preparation: electrical membrane properties and response characteristics to subthalamic stimulation, Brain Research, 437 (1987) 45-55.

35 Napier, T.C., Pirch, J.H. and Peterson, S.L., Spontaneous unit activity in the globus pallidus following cumulative injections of morphine in phenobarbital or chloral hydrateanesthetized rats, Neuropharmacology, 22 (1983) 165-171.

36 Napier, T.C., Pirch, J.H. and Strahlendorf, H.K., Naloxone antagonizes striatally-induced suppression of globus pallidus unit activity, Neuroscience, 9 (1983) 53-59.

37 Nauta, H.J.W., Projections of the pallidal complex: an autoradiographic study in the cat, Neuroscience, 4 (1979) 1853-1873.

38 Niimi, K., Ikeda, T., Kawamura, S. and Inoshita, H., Efferent projections of the head of the caudate nucleus in the cat, Brain Research, 21 (1970) 327-343.

39 Obata, K. and Yoshida, M., Caudate-evoked inhibition and actions of GABA and other substances on cat pallidal neurons, Brain Research, 64 (1973) 455-459.

40 Oertel, W.H., Schmechel, D.E., Brownstein, M.J., Tappaz, M.L., Ransom, D.H. and Kopin, I.J., Decrease of 
glutamate decarboxylase (GAD)-immunoreactive terminals in the substantia nigra after kainic acid lesion of the striatum, J. Histochem. Cytochem., 29 (1980) 977-980.

41 Okoyama, S., Nakamura, Y., Kitao, Y. and Moriizumi, T., The synaptic organization of the cat globus pallidus: a combined electron microscopic degeneration and HRP study, Neurosci. Lett., Suppl. 17 (1984) S76.

42 Pan, H.S., Frey, K.A., Young, A.B. and Penney, J.B., Changes in $\left[{ }^{3} \mathrm{H}\right]$ muscimol binding in substantia nigra, entopeduncular nucleus, globus pallidus and thalamus after striatal lesions as demonstrated by quantitative receptor autoradiography, J. Neurosci., 3 (1983) 1189-1198.

43 Parent, A., Comparative Neurobiology of the Basal Ganglia, Wiley, New York, NY, 1986.

44 Park, M.R., Falls, W.M. and Kitai, S.T., An intracellular HRP study of the rat globus pallidus. I. Responses and light microscopic analyses, J. Comp. Neurol., 211 (1984) 284-294.

45 Pearson, R.C.A., Neal, J.W. and Powell, T.P.S., Bilateral morphological changes in the substantia nigra of the rat following unilateral damage of the striatum, Brain Research, 400 (1987) 127-132.

46 Penney, J.B. and Young, A.B., Striatal inhomogeneities and basal ganglia function, Movement Disorders, 1 (1986) 3-15.

47 Penney, J.B. and Young, A.B., Speculations on the functional anatomy of basal ganglia disorders, Ann. Rev. Neurosci., 6 (1983) 73-94.

48 Penny, G.R., Afsharpour, S. and Kitai, S.T., The glutamate decarboxylase-, leucine enkephalin-, methionine enkephalin- and substance P-immunoreactive neurons in the neostriatum of the rat and cat: evidence for partial population overlap, Neuroscience, 17 (1986) 1011-1045.

49 Perkel, D.H. and Bullock, T.H., Neural Coding, Neurosci. Res. Prog. Bull., 6 (1968) 221-343.

50 Pinnock, R.D., Woodruff, G.N. and Turnbull, M.J., Actions of substance P, MIF, TRH and related peptides in the substantia nigra, caudate nucleus and nucleus accumbens, Neuropharmacology, 22 (1983) 687-696.

51 Ribak, C.E., Vaughn, J.E. and Roberts, E., The GABA neurons and their axon terminals in the rat corpus striatum as demonstrated by GAD immunocytochemistry, J. Comp. Neurol., 187 (1979) 261-284.

52 Robertson, R.G., Farmery, S.M., Sambrook, M.A. and Crossman, A.R., Dyskinesia in the primate following injection of an excitatory amino acid antagonist into the medial segment of the globus pallidus, Brain Research, 476 (1989) 317-322.

53 Royce, G.J. and Lane, E.J., Efferent connections of the caudate nucleus, including cortical projections of the striatum and other basal ganglia: an autoradiographic and horseradish peroxidase investigation in the cat, J. Comp. Neurol., 226 (1984) 28-49.

54 Sachdev, R.N.S. and Aldridge, J.W., Effect of striatal lesions on spontaneous unit activity recorded from the globus pallidus, entopeduncular nucleus, and ventral pallidum, Soc. Neurosci. Abstr., 13 (1987) 981.

55 Sachdev, R.N.S., Gilman, S. and Aldridge, J.W., Bursting properties of units in cat globus pallidus and entopeduncular nucleus: the effect of excitotoxic striatal lesions, submitted.

56 Sirinathsinghji, D.J.S., Dunnett, S.B., Isacson, O., Clarke, D.J., Kendrick, K. and Björklund, A., Striatal grafts in rats with unilateral neostriatal lesions - II. In vivo monitoring of GABA release in globus pallidus and substantia nigra, Neuroscience, 24 (1988) 803-811.

57 Snider, R.S. and Neimer, W.T., A Stereotaxic Atlas of the Cat Brain, University of Chicago Press, Chicago, IL, 1961.

58 Steward, O., Lesion-induced synapse growth in the hippocampus. In K.H. Pribram and R.L. Issacson (Eds.), The Hippocampus, Vol. 3, Plenum, New York, 1986, pp. $65-111$.

59 Thompson, P.D., Berardelli, A., Rothwell, J.C., Day, B.L., Dick, J.P.R., Benecke, R, and Marsden, C.D., The coexistence of bradykinesia and chorea in Huntington's disease and its implications for theories of basal ganglia control of movement, Brain, 111 (1988) 223-244.

60 Vibert, J.F. and Costa, J., Spike separation in multiunit records: a multivariate analysis of spike descriptive parameters, EEG Clin. Neurophysiol., 47 (1979) 172-182.

61 Yelnik, J., Percheron, G. and Francois, C., A Golgi analysis of the primate globus pallidus. II. Quantitative morphology and spatial orientation of dendritic arborizations, J. Comp. Neurol., 227 (1984) 200-213.

62 Yip, V.S., Zhang, W.P., Woolsey, T.A. and Lowry, O.H., Quantitative histochemical and microchemical changes in the adult mouse central nervous after section of the infraorbital and optic nerves, Brain Research, 406 (1987) $157-170$.

63 Zaczek, R. and Coyle, J.T., Excitatory amino acid analogues: neurotoxicity and seizures, Neuropharmacology, 21 (1982) 15-26. 